\title{
Prevalência de competições terapêuticas na farmacoterapia de usuários das Unidades de Saúde da Família de Ribeirão Preto, São Paulo
}

\author{
Prevalence of therapeutic competitions in pharmacotherapy of users of Family Health Units in \\ Ribeirão Preto, São Paulo \\ Prevalencia de competencias terapéuticas en farmacoterapia de usuarios de Unidades de Salud de \\ la Familia en Ribeirão Preto, São Paulo
}

Recebido: 20/04/2021 | Revisado: 27/04/2021 | Aceito: 01/05/2021 | Publicado: 15/05/2021

\author{
Anelize Roveri Arcanjo \\ ORCID: https://orcid.org/0000-0002-5504-5762 \\ Universidade de São Paulo, Brasil \\ E-mail: anelizearcanjo@gmail.com \\ Rinaldo Eduardo Machado de Oliveira \\ ORCID: https://orcid.org/0000-0003-1684-1456 \\ Universidade de São Paulo, Brasil \\ E-mail: rinaldo.eduardo@usp.br \\ Karoline Fonseca Costa \\ ORCID: https://orcid.org/0000-0002-0075-5118 \\ Universidade de São Paulo, Brasil \\ E-mail: karoline.costa@alumni.usp.br \\ Mayara Barbosa Pieroni \\ ORCID: https://orcid.org/0000-0001-6670-7605 \\ Universidade de São Paulo, Brasil \\ E-mail: mayarabpieroni@gmail.com \\ Julieta Ueta \\ ORCID: https://orcid.org/0000-0003-2645-0432 \\ Universidade de São Paulo, Brasil \\ E-mail: julietapurame@gmail.com
}

\begin{abstract}
Resumo
No atual cenário epidemiológico verifica-se elevada frequência de doenças crônicas não transmissíveis com o aumento no uso de medicamentos e competições terapêuticas. Assim, o objetivo deste estudo foi estimar a prevalência e identificar as competições terapêuticas em usuários que faziam seguimento em seis Unidades de Saúde da Família de Ribeirão Preto, São Paulo. Trata-se de um estudo retrospectivo, longitudinal, descritivo e exploratório com amostra constituída por 226 participantes. A maioria era idoso (69,5\%), pertencia ao sexo feminino (63,7 \%), era sedentário $(48,2 \%)$ e apresentava quatro ou cinco doenças crônicas não transmissíveis $(50,8 \%)$, sendo mais frequentes a hipertensão arterial sistêmica, o diabetes mellitus tipo 2 e dislipidemia. O risco potencial de ocorrência de competições terapêuticas foi estimado em 64,2\%. Dentre elas, destacou-se a competição terapêutica que envolvia os betabloqueadores, utilizados para o tratamento da hipertensão arterial. Os dados evidenciam a necessidade de uma abordagem integral no cuidado às pessoas com multimorbidades na Atenção Primária à Saúde. Além disso, alerta os riscos das competições terapêuticas e a importância do monitoramento das complicações da doença índice na presença de uma competição terapêutica potencial.
\end{abstract}

Palavras-chave: Uso de medicamentos; Multimorbidade; Atenção primária à saúde.

\begin{abstract}
In the current epidemiological scenario, there is a high frequency of chronic non-communicable diseases with the increase in the use of drugs and therapeutic competitions. Thus, the aim of this study was to estimate the prevalence and identify therapeutic competitions in users who were followed up in six Family Health Units in Ribeirão Preto, São Paulo. This is a retrospective, longitudinal, descriptive and exploratory study with a sample consisting of 226 participants. Most were elderly (69.5\%), belonged to the female sex $(63.7 \%)$, were sedentary $(48.2 \%)$ and had four or five chronic non-communicable diseases $(50.8 \%)$, the most frequent being systemic arterial hypertension. The potential risk for the occurrence of therapeutic competitions was estimated at $64.2 \%$. Among them, therapeutic competition between betablockers, used for the treatment of arterial hypertension, and type 2 diabetes mellitus stood out. The data show the need for a comprehensive approach in the care of the individual with multimorbidities in Primary Health Care. In addition, it alerts the risks of therapeutic competitions and the importance of monitoring the complications of the index disease in the presence of potential therapeutic competition.
\end{abstract}


Keywords: Drug utilization; Multimorbidity; Primary health care.

\section{Resumen}

En el escenario epidemiológico actual, existe una alta frecuencia de enfermedades crónicas no transmisibles con el aumento del uso de medicamentos y competencias terapéuticas. Así, el objetivo de este estudio fue estimar la prevalencia e identificar competencias terapéuticas en usuarios que fueron seguidos en seis Unidades de Salud de la Familia en Ribeirão Preto, São Paulo. Se trata de un estudio retrospectivo, longitudinal, descriptivo y exploratorio con una muestra de 226 participantes. La mayoría eran ancianos (69,5\%), pertenecían al sexo femenino (63,7\%), eran sedentarios $(48,2 \%)$ y tenían cuatro o cinco enfermedades crónicas no transmisibles $(50,8 \%)$, siendo las más frecuentes la hipertensión arterial sistémica, la diabetes tipo 2 mellitus y dislipidemia. El riesgo potencial de aparición de competencias terapéuticas se estimó en $64,2 \%$. Entre ellos, destacó la competencia terapéutica entre los betabloqueantes, utilizados para el tratamiento de la hipertensión arterial. Los datos muestran la necesidad de un abordaje integral en la atención del individuo con multimorbilidades en Atención Primaria de Salud, alerta los riesgos de las competencias terapéuticas y la importancia de monitorear las complicaciones de la enfermedad índice en presencia de una competencia terapéutica potencial.

Palabras clave: Utilización de medicamentos; Multimorbilidad; Atención primaria de salud.

\section{Introdução}

As doenças crônicas não transmissíveis (DCNTs) constituem um dos maiores problemas de saúde pública no mundo e estão relacionadas a quatro principais fatores de risco modificáveis: o tabagismo, a inatividade física, o uso prejudicial do álcool e alimentação não saudável (WHO, 2005). Traduzem-se em elevado número de mortes prematuras e perda da qualidade de vida, com alto grau de limitação e incapacidades para as atividades de vida diária (Malta et al., 2013). As DCNTs são caracterizadas por apresentarem etiologia múltipla, longos períodos de latência e curso prolongado (Malta et al., 2017). O declínio da taxa de mortalidade e o aumento da expectativa de vida devido a avanços técnicos e científicos nas condições de saúde da população expõem a mesma a um maior risco de desenvolvimento de doenças. (Oliveira-Campos et al., 2013). Dessa forma, há um aumento na prevalência das DCNTs e consequentemente de multimorbidades, com indivíduos acometidos de diversas enfermidades simultaneamente e uso contínuo de um elenco de medicamentos.

A multimorbidade consiste na ocorrência de duas ou mais condições crônicas físicas ou mentais simultaneamente em um indivíduo. Sua prevalência está relacionada ao aumento da idade e ao sexo feminino. (Salive, 2013; Fortin, Stewart, Poitras, Almirall \& Maddocks, 2012). O início precoce da multimorbidade está associado ao baixo nível socioeconômico, sendo cada vez mais comum em países de baixa e média renda (MacMahon FMedSci, Elliott FMedSci \& Levitt, 2018). Embora o risco de multimorbidade aumente com o avançar da idade, ela não é exclusiva em idosos, sendo comum também em jovens e em indivíduos de meia idade (MacMahon \& The Academy of Medical Sciences, 2018).

O elevado número de doenças aumenta o risco de uma farmacoterapia inadequada, com maior possibilidade de polifarmácia, baixa adesão à farmacoterapia, reações e eventos adversos, competições terapêuticas (CTs) e interações entre tratamentos para diferentes problemas de saúde, bem como para um mesmo problema de saúde (Ickowicz, 2012). Em pessoas com multimorbidade, geralmente são prescritos vários medicamentos para tratar suas condições individualmente. Neste cenário, torna-se comum as CTs que são compreendidas como interações medicamento-doença, em que a farmacoterapia recomendada para uma condição de saúde pode afetar negativamente a outra condição coexistente. É necessário um medicamento (medicamento que compete), indicado para uma condição de saúde (doença índice) que interfere negativamente com outra doença coexistente (doença que compete) (Lorgunpai et al., 2014; Dumbreck et al., 2015; Molino, 2018).

Alguns casos estudados de CTs envolvem os inibidores de COX-2, indicados para a artrite, que influem negativamente nas doenças cardíacas (Graham, 2006), e a rosiglitazona para o tratamento do diabetes mellitus tipo 2 (DM2), que aumenta o risco de eventos cardiovasculares, interferindo assim, na insuficiência cardíaca (Steven, Nissen \& Kathy Wolski, 2007; Winkelmayer, Setoguchi, Levin \& Solomon, 2008). Destaca-se também como CT a interação entre os betabloqueadores não seletivos, indicados em algumas situações para a hipertensão arterial sistêmica (HAS), e a doença pulmonar obstrutiva 
crônica (DPOC). Esses medicamentos têm o potencial de exacerbar a DPOC pelo bloqueio no receptor $\beta 2$, aumentando assim, a obstrução brônquica e a reatividade da via aérea (Molino, 2018; Chang, Mills, McLachlan, Karalus \& Hancox, 2010).

Aproximadamente 75\% dos adultos mais velhos com múltiplas DCNTs estão em risco de CTs (Lorgunpai et al., 2014). Estudos sobre as CTs para embasar as decisões clínicas do cuidado de idosos com multimorbidades na Atenção Primária à Saúde (APS) ainda são escassos e a compreensão do real impacto na tomada de decisão clínica dos profissionais da saúde, principalmente do profissional prescritor ainda é limitado (Salive, 2013; Guthrie, Payne, Alderson, McMurdo \& Mercer, 2012; Onder et al., 2015). Assim, este estudo objetivou estimar a prevalência e identificar as CTs em usuários que faziam seguimento em seis Unidades de Saúde da Família (USFs) de Ribeirão Preto, São Paulo (SP).

\section{Metodologia}

Trata-se de um estudo retrospectivo, longitudinal, descritivo e exploratório sendo utilizados como fonte de dados secundários as evoluções clínicas, as prescrições de medicamentos e os resultados dos exames laboratoriais disponíveis no sistema eletrônico Hygia e prontuários individuais físicos das primeiras consultas dos anos de 2017-2019.

Foram incluídas nesse estudo pessoas de ambos os sexos, com idade maior ou igual a 20 anos, acompanhados em seis USFs, no distrito sanitário oeste do município de Ribeirão Preto, SP. Excluíram-se os indivíduos que eram cadastrados nas USFs mas faziam seguimento clínico em outros serviços de saúde e aqueles que não usavam medicamentos.

$\mathrm{Na}$ coleta de dados, considerou-se as variáveis sociodemográficas; antropométricas, tais como peso, altura e índice de massa corporal (IMC); clínicas; laboratoriais, resultado de aferição da pressão arterial, exames do perfil lipídico, glicemia em jejum e pós-prandial, hemoglobina glicada (HbA1c), creatinina, ureia, enzima creatinofosfoquinase (CPK), ácido úrico, enzimas alanina aminotransferase (ALT) e aspartato aminotransferase (AST), bem como, as variáveis relacionadas à farmacoterapia. Os dados foram coletados em um formulário semiestruturado e inseridos em planilhas do Excel. Posteriormente, construiu-se um banco de dados com a introdução de codificações.

$\mathrm{Na}$ análise das multimorbidades, todas as condições crônicas foram incluídas e foi considerada como a presença de duas ou mais condições crônicas de saúde simultâneas em um indivíduo. Já a identificação das CTs potenciais foi realizada de acordo com as pesquisas de Molino (2018) e Lorgunpai et al. (2014).

$\mathrm{Na}$ análise estatística, as variáveis categóricas foram analisadas por meio do cálculo de frequências absolutas e relativas, com intervalo de confiança de $95 \%$ (IC95\%) e as variáveis contínuas por média e desvio padrão, conforme apropriado. O banco de dados foi analisado pelo software $\mathrm{R}^{\circledR}$.

O presente estudo foi aprovado pelo Comitê de Ética em Pesquisa do Centro de Saúde Escola da Faculdade de Medicina de Ribeirão Preto da Universidade de São Paulo, mediante parecer nº 2833214 e CAAE nº 60902416.7.0000.5414 e com dispensa da aplicação do Termo de Consentimento Livre e Esclarecido (TCLE) pelo fato de empregar-se somente dados secundários na pesquisa.

\section{Resultados}

Foram empregados dados de 226 participantes que realizaram acompanhamento em seis USFs no período de 2017 a 2019. De acordo com a Tabela 1, na amostra estudada, $63,7 \%$ dos participantes pertenciam ao sexo feminino. A faixa etária variou de 36 a 91 anos, com média de 65,5 anos ( $\mathrm{DP}=12,6$ ), sendo que, 68,6 \% eram idosos (idade igual ou superior a 60 anos). Dentre os fatores de risco para as DCNTs, o sedentarismo foi mais prevalente, seguido do uso de álcool e tabagismo, respectivamente. Estes dados foram autorreferidos pelos usuários durante a anamnese das consultas médicas nas USFs. 
Research, Society and Development, v. 10, n. 5, e51910515256, 2021

(CC BY 4.0) | ISSN 2525-3409 | DOI: http://dx.doi.org/10.33448/rsd-v10i5.15256

Tabela 1. Características demográficas e socioeconômicas da amostra estudada. Ribeirão Preto -SP, 2017-2019. (n=226)

\begin{tabular}{|c|c|c|c|c|}
\hline Variável & Categoria & $\mathbf{n}$ & $\%$ & $\mathrm{IC}^{2} \% \%^{1}$ \\
\hline \multirow[t]{2}{*}{ Sexo } & Feminino & 144 & 63,7 & $57,4-70,0$ \\
\hline & Masculino & 82 & 36,3 & $30,0-42,6$ \\
\hline \multirow[t]{3}{*}{ Tabagismo $^{2}$} & Sim & 25 & 11,1 & $7,0-15,2$ \\
\hline & Não & 125 & 55,3 & $48,8-61,8$ \\
\hline & $\mathrm{NC}^{3}$ & 76 & 33,6 & $27,5-39,8$ \\
\hline \multirow[t]{3}{*}{ Uso de álcool² } & Sim & 42 & 18,6 & $13,5-23,7$ \\
\hline & Não & 93 & 41,1 & $34,7-47,6$ \\
\hline & $\mathrm{NC}^{3}$ & 91 & 40,2 & $36,9-46,7$ \\
\hline \multirow[t]{3}{*}{ Sedentarismo $^{2}$} & Sim & 109 & 48,2 & $41,7-54,7$ \\
\hline & Não & 31 & 13,7 & $9,2-18,2$ \\
\hline & $\mathrm{NC}^{3}$ & 86 & 38,0 & $31,7-44,4$ \\
\hline
\end{tabular}

${ }^{1}$ IC95\%: Intervalo de Confiança de $95 \%$. ${ }^{2}$ Relatado nos prontuários dos participantes do estudo. ${ }^{3} \mathrm{NC}$ : Não consta no prontuário. Fonte: Autores.

Conforme apresentado na Tabela 2 as DCNTs mais frequentes foram: HAS, DM2, dislipidemia, osteoartrose, hipotireoidismo e transtorno depressivo maior.

Tabela 2. Distribuição de doenças na amostra estudada. Ribeirão Preto - SP, 2017-2019. (n=998).

\begin{tabular}{|c|c|c|c|}
\hline Doença & $\mathbf{n}$ & $\%$ & IC95\% 1 \\
\hline Hipertensão Arterial Sistêmica & 208 & 20,8 & $18,3-23,4$ \\
\hline Diabetes Mellitus tipo 2 & 204 & 20,4 & $17,9-22,9$ \\
\hline Dislipidemia & 185 & 18,5 & $16,1-20,9$ \\
\hline Doenças Psiquiátricas & 65 & 6,5 & $5,0-8,0$ \\
\hline Osteoartrose & 36 & 3,6 & $2,5-4,8$ \\
\hline Hipotireoidismo & 35 & 3,5 & $2,4-46$ \\
\hline Doença Renal Crônica & 30 & 3,0 & $1,9-4,1$ \\
\hline Insuficiência Cardíaca & 26 & 2,6 & $1,6-3,6$ \\
\hline Acidente Vascular Cerebral & 12 & 1,2 & $0,5-1,9$ \\
\hline Neoplasias & 9 & 0,9 & $0,3-1,5$ \\
\hline Doença Pulmonar Obstrutiva Crônica & 9 & 0,9 & $0,3-1,5$ \\
\hline Hiperplasia Prostática Benigna & 8 & 0,8 & $0,2-1,4$ \\
\hline
\end{tabular}




\begin{tabular}{|c|c|c|c|}
\hline Infarto Agudo do Miocárdio & 6 & 0,6 & $0,1-1,1$ \\
\hline Doença de Alzheimer & 6 & 0,6 & $0,1-1,1$ \\
\hline Asma & 6 & 0,6 & $0,1-1,1$ \\
\hline Osteopenia & 5 & 0,5 & $0,1-0,9$ \\
\hline Osteoporose & 5 & 0,5 & $0,1-0,9$ \\
\hline Outras & 146 & 14,6 & $12,4-16,8$ \\
\hline
\end{tabular}

${ }^{1}$ IC95\%: Intervalo de Confiança de 95\%. Fonte: Autores.

A presença de multimorbidade foi observada em 223 participantes deste estudo. A maioria deles (50,8\%) apresentou quatro ou cinco DCNTs conforme apresentado na Tabela 3. As multimorbidades mais elencadas envolveram as DCNTs de maior frequência.

Tabela 3. Número de doenças na amostra estudada. Ribeirão Preto - SP, 2017-2019. (n=226)

\begin{tabular}{cccc}
\hline Número de doenças & $\mathbf{n}$ & $\%$ & IC95\% $^{\mathbf{1}}$ \\
\hline 1 & 3 & 1,3 & $0,0-2,8$ \\
2 & 16 & 7,1 & $3,7-10,4$ \\
3 & 41 & 18,1 & $23,3-3,23,2$ \\
4 & 66 & 29,2 & $16,3-27,1$ \\
5 & 49 & 21,7 & $9,2-18,2$ \\
6 & 31 & 13,7 & $3,4-9,9$ \\
\hline
\end{tabular}

${ }^{1}$ IC95\%: Intervalo de Confiança de 95\%. Fonte: Autores.

A prevalência estimada de CTs foi de 64,2\% entre os participantes do estudo, sendo que, 66,7\% dos indivíduos possuíam uma CT e 33,3\%, duas ou mais. Dos indivíduos com uma CT potencial, 63,8\% apresentaram idade igual ou superior a 60 anos. Já os indivíduos com duas ou mais CTs, 86,2\% eram idosos. A Tabela 4 apresenta o número de CTs potenciais. 
Tabela 4. Número de competições terapêuticas potenciais na amostra estudada. Ribeirão Preto - SP, 2017-2019. (n=87).

\begin{tabular}{cccc}
\hline $\begin{array}{c}\text { Número de competições } \\
\text { terapêuticas }\end{array}$ & $\mathbf{n}$ & $\%$ & IC95\% $^{\mathbf{1}}$ \\
\hline 1 & 58 & 66,7 & $56,8-76,6$ \\
2 & 15 & 17,2 & $2,3-13,8$ \\
3 & 7 & 8,0 & $0,2-9,0$ \\
4 & 4 & 4,6 & $0,0-3,4$ \\
5 & 1 & 1,1 & $0,0-3,4$ \\
9 & 1 & 1,1 & $0,0-3,4$ \\
\hline
\end{tabular}

${ }^{1}$ IC95\%: Intervalo de Confiança de 95\%. Fonte: Autores.

$\mathrm{Na}$ análise da farmacoterapia evidenciou-se vinte e dois tipos diferentes de CTs, sendo que, as principais delas estão descritas na Tabela 5.

Tabela 5. Competições terapêuticas mais frequentes na amostra estudada. Ribeirão Preto - SP, 2017-2019. (n=145).

\begin{tabular}{|c|c|c|c|c|c|}
\hline Doença Índice & Doença que compete & Medicamento que compete & $\mathbf{n}$ & $\%$ & IC95\% ${ }^{1}$ \\
\hline HAS & DM2 & $\beta$-bloqueadores & 54 & 37,2 & $29,4-45,1$ \\
\hline Depressão & Hipercolesterolemia & Antipsicóticos & 11 & 7,6 & $3,3-11,9$ \\
\hline Insuficiência Cardíaca & DM2 & $\alpha \beta$-bloqueadores & 10 & 6,9 & $2,8-11,0$ \\
\hline Depressão & $\mathrm{DC}$ & Antidepressivos tricíclicos & 6 & 4,1 & $0,9-7,4$ \\
\hline Depressão & DM2 & Antidepressivos tricíclicos & 5 & 3,4 & $0,5-6,4$ \\
\hline Depressão & HAS & Antidepressivos tricíclicos & 5 & 3,4 & $0,5-6,4$ \\
\hline Depressão & HAS & IRSN & 5 & 3,4 & $0,5-6,4$ \\
\hline Depressão & DM2 & Antipsicóticos & 4 & 2,8 & $0,1-5,4$ \\
\hline Asma & DM2 & Corticoides inalatórios & 4 & 2,8 & $0,1-5,4$ \\
\hline Depressão & $\mathrm{DC}$ & ISRS & 4 & 2,1 & $0,0-4,3$ \\
\hline Outras & & & 37 & 25,5 & $18,4-32,6$ \\
\hline
\end{tabular}

${ }^{1}$ IC95\%: Intervalo de Confiança de $95 \%$.

DC: doença cardiovascular; DM2: diabetes mellitus tipo 2; HAS: hipertensão arterial sistêmica; IRSN: inibidores de recaptação de serotonina e norepinefrina; ISRS: inibidores seletivos de recaptação de serotonina.

Fonte: Autores. 


\section{Discussão}

Neste estudo, verificou-se predomínio de indivíduos com idade igual ou superior a 60 anos e do sexo feminino. Este fato pode estar associado a uma procura maior das mulheres ao sistema de saúde, em comparação aos homens, devido a sua maior tendência ao autocuidado, à medida que envelhecem (Andrade et al., 2015).

Em relação aos fatores de risco para as DCNTs, o sedentarismo foi o que obteve maior frequência. Considerado um dos principais fatores de risco para o desenvolvimento de doenças crônicas, ele repercute negativamente na saúde física e mental, bem como na qualidade de vida. A atividade física insuficiente atinge aproximadamente $31 \%$ da população mundial (WHO, 2011), sendo a obesidade o terceiro fator de risco que causou o maior número de mortes no Brasil em 2017 (Roth et al., 2018).

A HAS foi a DCNT mais prevalente, com frequência de 20,8\%, comparável com a literatura, na qual apresenta uma frequência de 21,4\% (Malta et al., 2015). Ela acomete principalmente pessoas de maior idade, atingindo mais de 70\% das pessoas acima de 70 anos (MS, 2006). Por outro lado, foram encontradas frequências maiores de DM2, dislipidemia, hipotireoidismo e osteartrose.

Observou-se uma alta prevalência de multimorbidade, 98,7\%, sendo este dado superior ao verificado em estudos anteriores (Salive, 2013; Melo \& Lima, 2020; Nguyen, Ngangue, Haggerty, Bouhali \& Fortin, 2019). Além disso, uma revisão sistemática da literatura mostrou que a prevalência de multimorbidade em pessoas idosas pode variar de 55 a $98 \%$ (Marengoni et al.,2011). Aproximadamente 50\% dos indivíduos apresentaram quatro a cinco DCNTs. Isso pode ser explicado pelas características da amostra do estudo, isto é, indivíduos que apresentam condições crônicas de saúde, principalmente HAS e DM2.

É difícil chegar a um consenso sobre a prevalência da multimorbidade, pois está relacionada ao acesso a cuidados de saúde e taxas de diagnóstico de DCNTs na população. Além disso, a prevalência de multimorbidade pode ser maior dependendo da definição empregada e do número de condições de saúde incluídas nessa definição (Salive, 2013; MacMahon \& The Academy of Medical Sciences, 2018). Para esse estudo, a definição de multimorbidade considerada foram duas ou mais condições crônicas de saúde.

Evidenciou-se que 64,2\% dos indivíduos estão em risco potencial de CTs e que, 66,7\% dos indivíduos da amostra apresentaram o risco de ocorrência de pelo menos uma CT e 33,3\%, o risco de 2 ou mais. Em relação aos idosos, 80,6\% apresentaram o risco potencial de pelo menos uma CT. Esse resultado corrobora o estudo de Lorgunpai et al. (2014), em que aproximadamente $75 \%$ dos adultos mais velhos com múltiplas doenças crônicas estão em risco de competição terapêutica.

Verificou-se também um aumento no número de CT em relação aos indivíduos idosos (idade maior ou igual a 60 anos) sobre os não idosos (idade menor do que 60 anos), tanto para o risco potencial de uma CT quanto para duas ou mais CTs. Com o envelhecimento, há um aumento da prevalência de multimorbidade, polifarmácia e consequentemente problemas na farmacoterapia (Salive, 2013; Lorgunpai et al., 2014; Nguyen et al., 2019).

A CT de maior frequência foi entre os betabloqueadores, utilizados para o tratamento da HAS (doença índice) e DM2. No estudo de Lorgunpai et al. (2014) também foi a principal CT. A classe terapêutica dos betabloqueadores adrenérgicos envolve um conjunto de fármacos que possuem efeitos diferenciados nos diferentes receptores adrenérgicos. Os betabloqueadores adrenérgicos são utilizados na clínica para a HAS, doença cardíaca isquêmica, insuficiência cardíaca congestiva e certas arritmias. Esses agentes farmacológicos, embora apresentem em comum o bloqueio de receptores betaadrenérgicos, possuem perfis farmacológicos diferentes, incluindo seletividade nos receptores $\beta 1$ e $\beta 2$, lipossolubilidade e consequentemente, penetração no Sistema Nervoso Central, parâmetros farmacocinéticos e efeitos vasodilatadores associados (Brunton, Hilal-Dandan \& Knollmann, 2019). 
Os betabloqueadores da primeira geração apresentam como protótipo o propranolol e possuem afinidade igual para os receptores $\beta 1$ e $\beta 2$. Já o atenolol, o metopropolol e o bisoprolol são exemplos de fármacos da segunda geração e antagonistas $\beta 1$ seletivos, isto é, têm afinidade um pouco maior para $\beta 1$ do que para $\beta 2$, embora essa seletividade não seja absoluta. Além destes betabloqueadores, há fármacos que possuem ações vasodilatadoras, além do bloqueio dos receptores $\beta$ e constituem a terceira geração. Os efeitos vasodilatadores são produzidos por meio de uma variedade de mecanismos, incluindo o bloqueio do receptor $\alpha 1$ (labetalol, carvedilol, bucindolol, bevantolol, nipradilol), aumento da produção de óxido nítrico (celiprolol, nebivolol, carteolol, bopindolol, nipradolol), propriedades agonistas $\beta 2$ (celiprolol, carteolol, bopindolol), bloqueio de entrada de cálcio (carvedilol, betaxolol, bevantolol), abertura de canais potássio (tilisolol) e ação antioxidante (carvedilol) (Brunton, Hilal-Dandan \& Knollmann, 2019).

OS eventos adversos dos betabloqueadores dependem da especificidade pelo subtipo de receptor, de sua distribuição nos receptores $\beta$ adrenérgicos e de seu grau de solubilidade (Helfand, Peterson, Christensen, Dana \& Thakurata, 2009). Os principais eventos metabólicos, como intolerância à glicose, e consequentemente, interferência negativa no DM foram observados com os betabloqueadores mais antigos, que não apresentam ação vasodilatadora periférica, pois o aumento da resistência vascular diminui a disponibilidade de glicose e reduz seu uso pelo músculo esquelético, o que gera intolerância à glicose (Kveiborg, Christiansen, Major-Petersen \& Torp-Pedersen, 2006).

Outra CT observada nesta pesquisa e relatada no estudo de Molino (2018) foi entre os antipsicóticos, indicados, em algumas situações para o tratamento da depressão, com a dislipidemia. É desconhecido ainda o mecanismo exato das alterações indesejáveis nos níveis lipídicos ocasionadas pelos antipsicóticos, porém existe uma correlação desses fármacos com o aumento de peso e assim, com alterações no perfil lipídico. As alterações mais relevantes nos triglicerídeos e no low density lipoprotein ou lipoproteína de baixa densidade (LDL-c) estão associadas ao uso de clozapina e olanzapina, drogas que produzem o maior ganho ponderal. Desta forma, é necessário o monitoramento do perfil lipídico nos usuários em tratamento com antipsicóticos (Cerqueira Filho, Arandas, Oliveira \& Sena, 2006; Touger, 2006).

É importante salientar que a existência de uma CT não representa uma contraindicação ao uso de medicamentos, porém, enfatiza a importância de uma avaliação da farmacoterapia. É imprescindível o monitoramento das pessoas com risco de CTs em relação às complicações da doença índice, pela competição existente com um medicamento prescrito para outra condição de saúde coexistente (Molino, 2018). O estudo apresentou como limitação a ausência de dados nos prontuários e/ou sistema Hygia referentes aos hábitos alimentares, prática de exercício físico, eventos adversos observados ou relatados, histórico de doenças prévias e medicamentos prescritos. Em vista disso, ressalta-se se a importância do registro adequado destes dados por diferentes profissionais com a transcrição de todas as informações relacionadas ao cuidado das pessoas nas USFs.

As perspectivas possíveis para pesquisas futuras consistem em compreender o impacto das CTs potenciais na decisão clínica dos profissionais das USFs, especialmente do prescritor. Os estudos relacionados às CTs de multimorbidades tornam-se necessárias para qualificar o cuidado aos usuários dos serviços de saúde, principalmente os idosos. (Salive, 2013; Guthrie et al., 2012; Onder et al., 2015).

\section{Considerações Finais}

Neste estudo, evidenciou-se que $64,2 \%$ dos usuários das USFs estavam em risco potencial de CT. Destacou-se a CT entre os betabloqueadores, utilizados para o tratamento da HAS, e o DM2. Na amostra do estudo, $69,5 \%$ dos usuários eram idosos, $63,7 \%$ pertenciam ao sexo feminino; $48,2 \%$ eram sedentários; 50,8\% apresentaram de quatro a cinco DCNTs, sendo as mais prevalentes a HAS, o DM2 e a dislipidemia. Estes dados apontam para a necessidade de um novo olhar dos profissionais 
da saúde ao cuidado integral às pessoas com múltiplas doenças crônicas, rompendo com modelos assistenciais fragmentados e centrados em uma doença específica. Além disso, alerta os riscos de potenciais CTs na APS.

\section{Referências}

Andrade, S. S. A., Stopa, S. R., Brito, A. S., Chueri, P. S., Szwarcwald, C. L., \& Malta, D. C. (2015). Prevalência de hipertensão arterial autorreferida na população brasileira: análise da Pesquisa Nacional de Saúde, 2013. Epidemiologia e Serviços de Saúde, 24(2), 297-304. https://doi.org/10.5123/s167949742015000200012

Brunton, L.L., Hilal-Dandan R., \& Knollmann, B. C. (2019). Goodman \& Gilman: As Bases Farmacológicas da Terapêutica. Editora Artmed.

Cerqueira Filho, E. A., Arandas, F. D. S., Oliveira, I. R., \& Sena, E. P. (2006). Dislipidemias e antipsicóticos atípicos. Jornal Brasileiro de Psiquiatria, 55(4), 296-307. https://doi.org/10.1590/S0047-20852006000400006

Chang, C. L., Mills, G. D., McLachlan, J. D., Karalus, N. C., \& Hancox, R. J. (2010). Cardio-selective and non-selective beta-blockers in chronic obstructive pulmonary disease: Effects on bronchodilator response and exercise. Internal Medicine Journal, 40(3), 193-200. https://doi.org/10.1111/j.14455994.2009.01943.x

Dumbreck, S., Flynn, A., Nairn, M., Wilson, M., Treweek, S., Mercer, S. W., Alderson, P., Thompson, A., Payne, K., \& Guthrie, B. (2015). Drug-disease and drug-drug interactions: Systematic examination of recommendations in $12 \mathrm{UK}$ national clinical guidelines. BMJ (Online), 350, 1-8. https://doi.org/10.1136/bmj.h949

Fortin, M., Stewart, M., Poitras, M. E., Almirall, J., \& Maddocks, H. (2012). A systematic review of prevalence studies on multimorbidity: Toward a more uniform methodology. Annals of Family Medicine, 10(2), 142-151. https://doi.org/10.1370/afm.1337

Gnjidic, D., Hilmer, S. N., Blyth, F. M., Naganathan, V., Waite, L., Seibel, M. J., McLachlan, A. J., Cumming, R. G., Handelsman, D. J., \& Le Couteur, D. G. (2012). Polypharmacy cutoff and outcomes: Five or more medicines were used to identify community-dwelling older men at risk of different adverse outcomes. Journal of Clinical Epidemiology, 65(9), 989-995. https://doi.org/10.1016/j.jclinepi.2012.02.018

Graham, D. J. (2006). The Seduction of Common Sense. 20993(13), 1-4.

Guthrie, B., Payne, K., Alderson, P., McMurdo, M. E. T., \& Mercer, S. W. (2012). Adapting clinical guidelines to take account of multimorbidity. BMJ (Online), 345(7878), 1-5. https://doi.org/10.1136/bmj.e6341

Helfand, M., Peterson, K., Christensen, V., Dana, T., \& Thakurata, S. (2009). Drug Class Review Beta Adrenergic Blockers. Oregon Health and Science University, July, 1-616. http://derp.ohsu.edu/final/BB_Final_Evidence Tables_Update 4_09_JUL.pdf

Ickowicz, E. (2012). Guiding principles for the care of older adults with multimorbidity: An approach for clinicians: American Geriatrics Society expert panel on the care of older adults with multimorbidity. Journal of the American Geriatrics Society, 60(10). https://doi.org/10.1111/j.1532-5415.2012.04188.x

Kveiborg, B., Christiansen, B., Major-Petersen, A., \& Torp-Pedersen, C. (2006). Metabolic effects of $\beta$-adrenoceptor antagonists with special emphasis on carvedilol. American Journal of Cardiovascular Drugs, 6(4), 209-217. https://doi.org/10.2165/00129784-200606040-00001

Lorgunpai, S. J., Grammas, M., Lee, D. S. H., McAvay, G., Charpentier, P., \& Tinetti, M. E. (2014). Potential therapeutic competition in community-living older adults in the U.S.: Use of medications that may adversely affect a coexisting condition. PLoS ONE, 9(2). https://doi.org/10.1371/journal.pone.0089447

MacMahon FMedSci, S., Elliott FMedSci, P., \& Levitt, N. (2018). Advancing research to tackle multimorbidity: the UK and LMIC perspectives. June. https://acmedsci.ac.uk/file-download/11182404

MacMahon, S., \& The Academy of Medical Sciences. (2018). Multimorbidity: a priority for global health research. Academy of Medical Sciences, April. https://acmedsci.ac.uk/file-download/82222577

Malta, D. C., Stopa, S. R., Szwarcwald, C. L., Gomes, N. L., Silva Júnior, J. B., \& Reis, A. A. C. (2015). A vigilância e o monitoramento das principais doenças crônicas não transmissíveis no Brasil - pesquisa nacional de saúde, 2013. Revista Brasileira de Epidemiologia, 18, 3-16. https://doi.org/10.1590/1980- 5497201500060002

Malta, D. C., Bernal, R. T. I., Lima, M. G., Araújo, S. S. C., Silva, M. M. A., Freitas, M. I. F., \& Barros, M. B. de A. (2017). Doenças crônicas não transmissíveis e a utilização de serviços de saúde: análise da Pesquisa Nacional de Saúde no Brasil. Rev Saúde Publica, 51(1), 1-10. https://doi.org/10.1590/S1518- 8787.2017051000090

Marengoni, A., Angleman, S., Melis, R., Mangialasche, F., Karp, A., Garmen, A., Meinow, B., \& Fratiglioni, L. (2011). Aging with multimorbidity: A systematic review of the literature. Ageing Research Reviews, 10(4), 430-439. https://doi.org/10.1016/j.arr.2011.03.003

Melo, L. A., \& Lima, K. C. (2020). Factors associated with the most frequent multimorbidities in Brazilian older adults. Ciência e Saúde Coletiva, 25(10), 3879- 3888. https://doi.org/10.1590/1413-812320202510.35632018

Ministério da Saúde. Secretaria de Atenção à Saúde. Departamento de Atenção Básica. (2006). Envelhecimento e saúde da pessoa idosa. Brasília: Ministério da Saúde.

Molino, C.G.R.C (2018). Estudo da prevalência de competições terapêuticas entre idosos com multimorbidades do estudo SABE (Saúde, Bem-Estar e Envelhecimento. Dissertação de doutorado. Universidade de São Paulo, São Paulo, SP, Brasil. 
Research, Society and Development, v. 10, n. 5, e51910515256, 2021

(CC BY 4.0) | ISSN 2525-3409 | DOI: http://dx.doi.org/10.33448/rsd-v10i5.15256

Nguyen, T. N., Ngangue, P., Haggerty, J., Bouhali, T., \& Fortin, M. (2019). Multimorbidity, polypharmacy and primary prevention in community-dwelling adults in Quebec: A cross-sectional study. Family Practice, 36(6), 706-712. https://doi.org/10.1093/fampra/cmz023

Oliveira-Campos, M., Neto, J. F. R.-, Silveira, M. F., Neves, D. M. R., Vilhena, J. M., Oliveira, J. F., Magalhães, J. C., Drumond, D. (2013). The impact of risk factors of non-communicable chronic diseases on quality of life. Ciência e Saúde Coletiva, 18(3), 873-882. https://doi.org/10.1590/S141381232013000300033

Onder, G., Palmer, K., Navickas, R., Jurevičiene, E., Mammarella, F., Strandzheva, M., Mannucci, P., Pecorelli, S., \& Marengoni, A. (2015). Time to face the challenge of multimorbidity. A European perspective from the joint action on chronic diseases and promoting healthy ageing across the life cycle (JACHRODIS). European Journal of Internal Medicine, 26(3), 157-159. https://doi.org/10.1016/j.ejim.2015.02.020

Roth, G. A., Abate, D., Abate, K. H., Abay, S. M., Abbafati, C., Abbasi, N., Abbastabar, H., Abd-Allah, F., Abdela, J., Abdelalim, A., Abdollahpour, I., Abdulkader, R. S., Abebe, H. T., Abebe, M., Abebe, Z., Abejie, A. N., Abera, S. F., Abil, O. Z., Abraha, H. N., \& Murray, C. J. L. (2018). Global, regional, and national age-sex-specific mortality for 282 causes of death in 195 countries and territories, 1980-2017: a systematic analysis for the Global Burden of Disease Study 2017. The Lancet, 392(10159), 1736-1788. https://doi.org/10.1016/S0140-6736(18)32203-7

Salive, M. E. (2013). Multimorbidity in older adults. Epidemiologic Reviews, 35(1), 75-83. https://doi.org/10.1093/epirev/mxs009

Steven, E. Nissen M. D, \& Kathy Wolski, M. P. H. (2007) Effect of rosiglitazone on the risk of myocardial infarction and death from cardiovascular causes. The New England Journal of Medicine, 356, 2457-2471.

Touger J. (2006). Introductory physics: building understanding. Diabetes Care, 27(2), 904.

Winkelmayer, W. C., Setoguchi, S., Levin, R., \& Solomon, D. H. (2008). Comparison of cardiovascular outcomes in elderly patients with diabetes who initiated rosiglitazone vs pioglitazone therapy. Archives of Internal Medicine, 168(21), 2368-2375.https://doi.org/10.1001/archinte.168.21.2368

World Health Organization. (2005). Preventing chronic diseases a vital investment. Geneva: WHO.

World Health Organization. (2011).Global status report on noncommunicable diseases. Geneva:WHO. 